\title{
C-reactive protein promotes diabetic kidney disease in a mouse model of type 1 diabetes
}

\author{
F. Liu • H. Y. Chen • X. R. Huang - A. C. K. Chung • \\ L. Zhou • P. Fu • A. J. Szalai • H. Y. Lan
}

Received: 4 January 2011 / Accepted: 8 June 2011 / Published online: 9 July 2011

(C) Springer-Verlag 2011

\begin{abstract}
Aims/hypothesis Although C-reactive protein (CRP) has been implicated as a risk factor in diabetes, its pathogenic importance in diabetic kidney disease (DKD) remains unclear. The present study investigated the potential role of CRP in DKD.

Methods Diabetes was induced by streptozotocin in human $C R P$ transgenic and wild-type mice for assessment of kidney injury at 24 weeks by real-time PCR, immunohistochemistry and western blot analysis. In vitro, the pathogenic effect of CRP was investigated using human kidney tubular epithelial cells cultured with high glucose and/or CRP.

Results We found that CRP transgenic mice developed much more severe diabetic kidney injury than wild-type mice, as indicated by a significant increase in urinary albumin
\end{abstract}

F. Liu and H. Y. Chen contributed equally to this study.

Electronic supplementary material The online version of this article (doi:10.1007/s00125-011-2237-y) contains peer-reviewed but unedited supplementary material, which is available to authorised users.

H. Y. Chen · X. R. Huang • A. C. K. Chung • H. Y. Lan $(\bowtie)$ Department of Medicine and Therapeutics, and Li Ka Shing Institute of Health Sciences, Chinese University of Hong Kong, the Prince of Wales Hospital, NT,

Hong Kong, SAR, People's Republic of China

e-mail: hylan@cuhk.edu.hk

F. Liu $\cdot$ L. Zhou $\cdot$ P. Fu

Department of Nephrology,

West China Hospital of Sichuan University,

Chengdu, People's Republic of China

\section{A. J. Szalai}

Department of Medicine,

The University of Alabama at Birmingham,

Birmingham, AL, USA excretion and kidney injury molecule-1 abundance, enhanced infiltration of macrophages and T cells, and upregulation of pro-inflammatory cytokines (IL-1 $\beta, \mathrm{TNF} \alpha$ ) and extracellular matrix (collagen I, III and IV). Enhanced renal inflammation and fibrosis in $C R P$ transgenic mice was associated with upregulation of CRP receptor, CD32a, and over-activation of the TGF- $\beta /$ SMAD and nuclear factor $\mathrm{kB}$ signalling pathways. In vitro, CRP significantly upregulated pro-inflammatory cytokines (IL-1 $\beta, \mathrm{TNF} \alpha$, monocyte chemoattractant protein-1 [MCP-1]) and pro-fibrotic growth factors (TGF- $\beta 1$, connective tissue growth factor [CTGF]) via $\mathrm{CD} 32 \mathrm{a} / 64$. CRP was induced by high glucose, which synergistically promoted high glucose-mediated renal inflammation and fibrosis.

Conclusions/interpretation CRP is not only a biomarker, but also a mediator in DKD. Enhanced activation of TGF$\beta / S M A D$ and nuclear factor $K B$ signalling pathways may be the mechanisms by which CRP promotes renal inflammation and fibrosis under diabetic conditions.

Keywords CRP. Diabetic nephropathy Fibrosis . Inflammation - NF- $k B$ - TGF- $\beta /$ SMAD

$\begin{array}{ll}\text { Abbreviations } \\ \text { CRP } & \text { C-reactive protein } \\ \text { CTGF } & \text { Connective tissue growth factor } \\ \text { DKD } & \text { Diabetic kidney disease } \\ \text { HK-2 } & \text { Human kidney epithelial cells } \\ \text { KIM-1 } & \text { Kidney injury molecule-1 } \\ \text { MCP-1 } & \text { Monocyte chemoattractant protein-1 } \\ \text { NF-kB } & \text { Nuclear factor } \text { KB } \\ \text { TEC } & \text { Tubular epithelial cell line } \\ \text { Tg } & \text { Transgenic } \\ \text { UAE } & \text { Urinary albumin excretion } \\ \text { Wt } & \text { Wild-type }\end{array}$




\section{Introduction}

Diabetic kidney disease (DKD) is the major cause of end-stage renal disease and is associated with chronic low-grade inflammation and activation of the innate immune system [1-3]. In patients with diabetes, low grade inflammation is evidenced by increased plasma levels of several biomarkers of inflammation, including C-reactive protein (CRP) $[1,2,4,5]$. Emerging evidence suggests that CRP is associated with increased risk of cardiovascular disease and diabetes [6, 7]. In patients with type 1 or type 2 diabetes, elevated CRP levels are associated with an increase in microalbuminuria [5, 8-11]. Increasing evidence shows that inflammatory and metabolic factors associated with diabetes, such as high glucose, adipokines, modified lipoproteins and NEFA are related to blood levels of CRP and may trigger its production [11, 12]. Numerous clinical studies have demonstrated that CRP predicts the treatment outcomes in cardiovascular disease in diabetic and non-diabetic populations [6, 7, 13-15]. Indeed, treatment of diabetes with either glucose-lowering drugs such as insulin and peroxisome proliferator-activated receptor gamma, or with non-diabetes drugs including aspirin and statins has led to substantial reductions of CRP and other cardiovascular risk markers in several clinical trial studies $[6,7,13-15]$. Positive outcomes in these trials further strengthen the value and acceptance of CRP as a predictive biomarker of cardiovascular disease risk in patients with diabetes mellitus, even though the underlying reasons for the association of CRP with these diseases remains unknown.

In some studies, human CRP has been shown to promote atherosclerosis in ApoE-deficient mice and contributes to thrombosis $[16,17]$. Most recently, we also demonstrated that human CRP exacerbates hypertensive cardiac remodelling under high angiotensin II conditions [18]. However, the pathogenic role of CRP in the development of diabetic complications remains unknown. Is CRP just a biomarker or is it involved in the pathogenesis of DKD? If CRP is indeed causal, then what is the mechanism of its action in diabetic renal inflammation and fibrosis? These fundamental questions were investigated in the present study, using a mouse model of type $1 \mathrm{DKD}$ induced in human CRP transgenic (Tg) mice and in vitro using human kidney epithelial cells (HK-2).

\section{Methods}

Mouse model of streptozotocin-induced diabetes Type 1 diabetes was induced in genetically identical littermate human CRP $\mathrm{Tg}$ and wild-type (Wt) mice (C57BL/6 background, male, aged $12-14$ weeks, $28-35$ g) by i.p. injection of $50 \mathrm{mg} / \mathrm{kg}$ streptozotocin daily for five consecutive days, as recommended by the Animal Models of Diabetic Complications Consortium [19]. The generation and characterisation of human CRP Tg mice have been described previously [20]. Diabetes was induced in groups of eight mice. In addition, groups of six normal $C R P \mathrm{Tg}$ and $\mathrm{Wt}$ mice that had been administered sodium citrate buffer (instead of streptozotocin) were used as normal agematched controls. Mice were killed under anaesthesia with ketamine/xyline delivered i.p. at 24 weeks and the renal cortexes were collected by carefully removing the renal pelvis and medullar tissues. Cortexes were used for real-time PCR and western blot analysis, or fixed in methyl Carnoy's for histological and immunohistochemical analysis. The experimental protocol was approved by the Animal Experimentation Ethics Committee at The Chinese University of Hong Kong.

Measurement of blood glucose, blood pressure and urinary albumin excretion Fasting ( 6 h) blood glucose was determined weekly for the first 2 weeks then every 4 weeks thereafter, using a blood glucose meter (Optium Xceed Systems, Doncaster, VIC, Australia). Blood pressure was performed by mouse-tail plethysmography using a blood pressure analysis system (BP2000; Visitech Systems, Apex, $\mathrm{NC}$, USA) in conscious mice. Before disease induction (week 0) urine was collected for $16 \mathrm{~h}$ by placing mice in metabolism cages and urinary albumin excretion (UAE) assay was performed. The process was repeated after disease induction (weeks 2, 4, 8, 16, 20 and 24). Urinary albumin was measured by competitive ELISA according to the manufacturer's instructions (Exocell, Philadelphia, PA, USA), with results expressed as total urinary microalbumin.

Histology and immunohistochemistry Changes in renal morphology were examined in methyl Carnoy's-fixed, paraffin-embedded tissue sections $(4 \mu \mathrm{m})$ stained with periodic acid-Schiff's reagent. Immnunohistochemistry was performed on paraffin sections using a microwavebased antigen retrieval technique [21]. The antibodies used in the study were as follows: rat anti-mouse monoclonal antibody to macrophage F4/80 (Serotec, Oxford, UK); and rabbit polyclonal antibodies to (1) T cell CD3 (SP7) and kidney injury molecule-1 (KIM-1) (both from Abcam, Cambridge, UK), (2) collagens I, III and IV (Southern Biotech, Birmingham, AL, USA), and (3) IL- $1 \beta, \mathrm{TNF} \alpha, \mathrm{TGF}-\beta$, connective tissue growth factor (CTGF), phospho-SMAD2/3, SMAD2/3 (Santa Cruz Biotechnology, Santa Cruz, CA, USA), a phospho-nuclear factor $\mathrm{kB}(\mathrm{NFkB}) / \mathrm{p} 65$ (Ser276) subunit (Cell Signaling Technology, Beverly, MA, USA) and CRP receptor CD32a (R\&D Systems, Minneapolis, MN, USA). After incubation with appropriate secondary antibodies, sections were developed with diaminobenzidine to produce a brown colour and counterstained with haematoxylin.

Abundance of IL- $1 \beta$, TNF $\alpha$, TGF- $\beta$, CTGF and CD32a in the entire cortex (a cross-section of the kidney) was determined 
using a quantitative image analysis system (AxioVision 4; Carl Zeiss, Göttingen, Germany). Briefly, the examined cortex was outlined and positive staining identified. Then the percentage of positively stained area within the outlined area of interest was determined. The number of $\mathrm{F} 4 / 80+, \mathrm{CD}^{+}$, phosphoSMAD2/3+ and phospho-NFkB/p65+ cells was counted in 20 consecutive glomeruli and expressed as cells/glomerular cross-section (gcs), whereas positive cells in the tubulointerstitium were counted under high-power fields $(\times 40)$ by means of a $0.0625 \mathrm{~mm}^{2}$ graticule fitted in the eyepiece of the microscope and expressed as cells per square millimetre. Quantitative analysis of immunostaining was carried out on coded slides as previously described [22, 23].

Cell culture The tubular epithelial cell line (TEC), HK-2, was purchased from American Type Culture Collection (ATCC) and cultured in DMEM-F12 (Hyclone, Logan, UT, USA) supplemented with $10 \%$ (vol./vol.) FBS (Invitrogen Life Technologies, Gaithersburg, MD, USA). TECs were deprived of FBS for $24 \mathrm{~h}$ and then stimulated with D-glucose at normal $(5.5 \mathrm{mmol} / \mathrm{l})$ or high $(35 \mathrm{mmol} / \mathrm{l})$ levels in the presence or absence of recombinant human CRP $(10 \mu \mathrm{g} / \mathrm{ml}$; R\&D Systems, ) for up to $24 \mathrm{~h}$ or in the presence of $0,1,5,10 \mu \mathrm{g} / \mathrm{ml} \mathrm{CRP}$ for $6 \mathrm{~h}$ (the predetermined peak reaction time). To determine whether the effects of CRP and high glucose were additive, cells were cultured in the presence of either CRP $(10 \mu \mathrm{g} / \mathrm{ml})$, high glucose $(35 \mathrm{mmol} / \mathrm{l})$ or both in combination. To block CRP activity, antibodies ( $5 \mu \mathrm{g} / \mathrm{ml}$ ) specific for the CRP receptors CD32a and CD64 (R\&D Systems) were added to culture $1 \mathrm{~h}$ before addition of CRP $(10 \mu \mathrm{g} / \mathrm{ml})$. D-Mannitol $(35 \mathrm{mmol} / \mathrm{l})$ was used as an osmotic control throughout the experiments and at least three independent experiments were performed.

Real-time PCR Kidney and cultured cell total RNA was extracted using a kit (RNeasy; Qiagen, Valencia, CA, USA) according to the manufacturer's instructions and cDNA was synthesised. Real-time PCR was performed with a real-time PCR detector (Opticon 2; Bio-Rad, Hercules, CA, USA) using IQ SYBR green supermix reagent (Bio-Rad) as described previously [22]. The primers for mouse mRNA of collagen I, collagen III, Tgfb1, Ctgf, Illb, Tnf $\alpha$ (also known as Tnf) and Gapdh have been described elsewhere $[22,23]$, while other primers were as shown in ESM Table 1. The housekeeping gene Gapdh was used as an internal standard. The ratio of specific mRNA: Gapdh mRNA was calculated and is expressed as mean \pm SEM.

Measurement of CRP and cytokines Human CRP in culture supernatant fraction was measured using a kit (Quantikine human CRP ELISA; R\&D Systems). Protein level of cytokines (TNF $\alpha$ and TGF- $\beta_{1}$ ) in the culture supernatant fractions was also determined by ELISA. Monoclonal antibodies specific for TNF $\alpha$ and TGF- $\beta_{1}$ and standards (TNF $\alpha$ and TGF- $\beta_{1}$ ) were purchased from R\&D Systems.

Western blot analysis Proteins were extracted from renal tissue with RIPA lysis buffer and analysed by western blot as previously described [22, 23]. Briefly, after blocking nonspecific binding with $5 \%$ (wt/vol.) BSA, membranes were incubated overnight for $24 \mathrm{~h}$ at $4^{\circ} \mathrm{C}$ with primary antibodies against phosphorylated NF-KB/p65, phosphorylated $\mathrm{SMAD} 2 / 3$ or CRP receptor CD32a. After being washed, the membranes were then incubated with IRDye 800 conjugated secondary antibodies (Rockland Immunochemicals, Gilbertsville, PA, USA). The signals were detected with an Odyssey Infrared Imaging System (Li-COR Biosciences, Lincoln, NE, USA) and quantified with Image J (National Institutes of Health, Bethesda, MD, USA; http://rsbweb.nih. gov/ij/download.html, accessed 21 June 2011). The ratio for the protein examined was normalised against GAPDH and expressed as the mean \pm SEM.

Statistical analyses The data obtained are expressed as mean \pm SEM. Statistical analyses were performed using oneway ANOVA, followed by two-tailed Newman-Keuls post test (Prism 3.0; GraphPad Software, San Diego, CA, USA).

\section{Results}

Diabetic renal injury is exacerbated in CRP Tg mice Elevated plasma human CRP $(6,387 \pm 1,413 \mathrm{ng} / \mathrm{ml})$ was detected by ELISA at baseline in CRP Tg mice. After injection of multiple low doses of streptozotocin for five consecutive days, hyperglycaemia (13.9-19.4 mmol/l) developed in CRP Tg and Wt mice, whose blood glucose levels were not significantly different under fasting conditions over the entire 24-week study period (Fig. 1a). Also, no significant difference in blood pressure was found between the genotypes $(128 \pm 2.7 \mathrm{mmHg}$ in Wt vs $134 \pm 2.5 \mathrm{mmHg}$ in CRP Tg). Surprisingly, however, CRP Tg mice developed more severe diabetic kidney injury than $\mathrm{Wt}$ mice, as evidenced by a significant increase in UAE (Fig. 1b) and marked upregulation of Kim-1 mRNA expression and protein (Fig. 1c-e). Histologically, diabetic Wt mice (at week 24 after streptozotocin injection) had bigger glomeruli, with hypercellularity and increased mesangial matrix, thickening of the glomerular basement membrane and tubulointerstitial extracellular matrix accumulation, than age- and genotype-matched saline-treated controls; these changes were further enhanced in CRP $\mathrm{Tg}$ mice (Fig. 1f, g).

Renal inflammation is exacerbated in diabetic CRP Tg mice As inflammation is a critical process in the development 


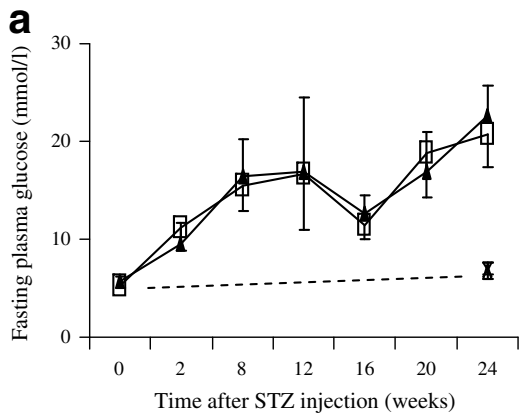

C

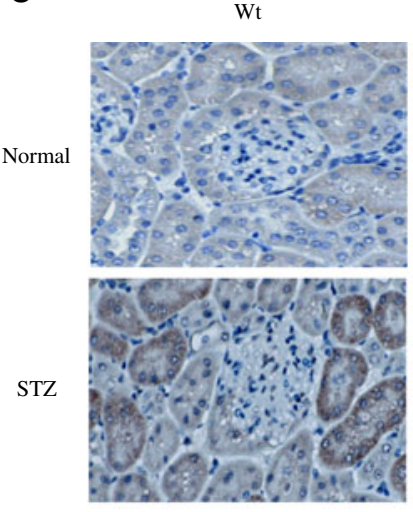

$\mathrm{Wt}$$$
\mathbf{f}
$$

\section{Normal}

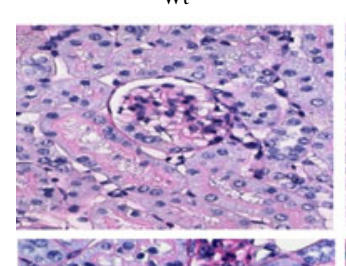

STZ
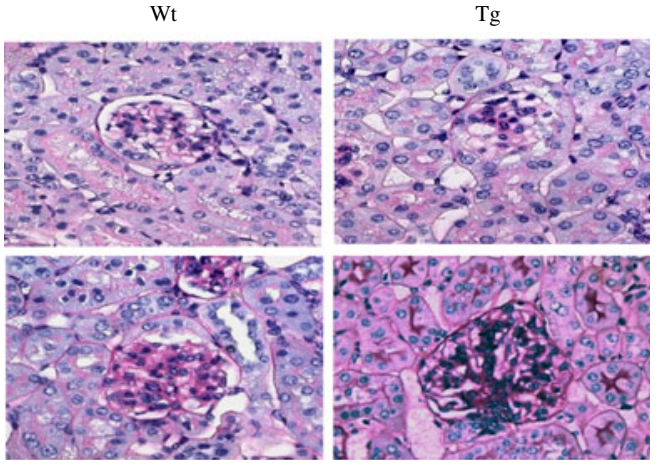

b

$\operatorname{Tg}$

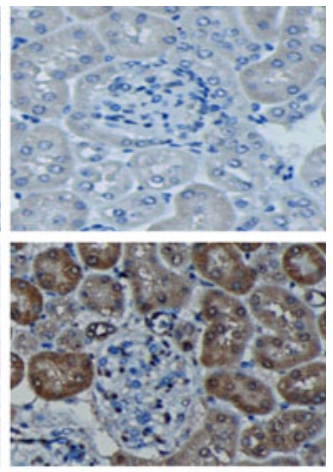

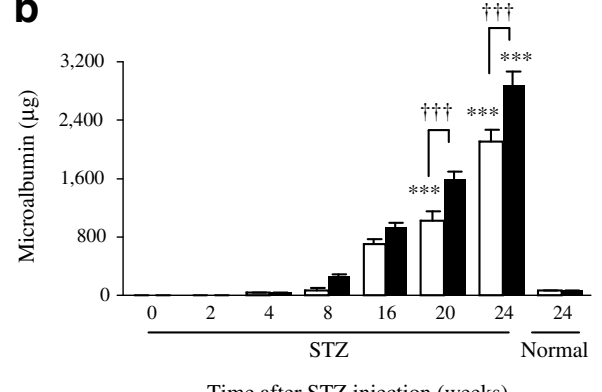

Time after STZ injection (weeks)

d
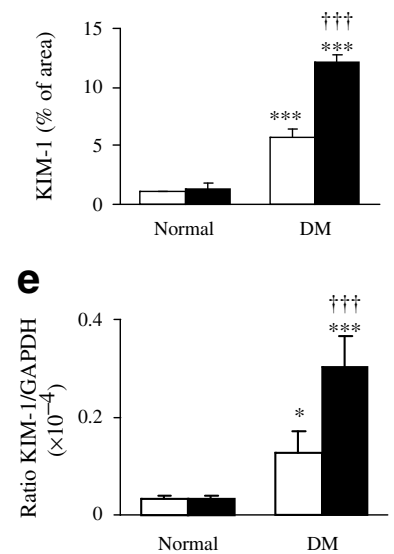

g

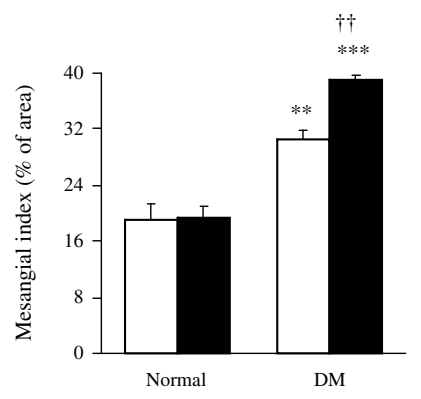

Fig. 1 Diabetic kidney injury is enhanced in $C R P$ Tg mice. a Fasting blood glucose after streptozotocin (STZ) treatment. In diabetic Wt (white squares) and CRP Tg (black triangles) mice, blood glucose levels were significantly increased and maintained at equal levels over the 24-week period. White triangles, non-diabetic Wt; cross symbol, non-diabetic Tg. b Diabetic CRP Tg (black bars) mice develop more severe microalbuminuria (UAE) than diabetic Wt (white bars) mice. c Immunohistochemistry and (d) quantitative analysis of KIM-1 in mice as above (b). DM, diabetes mellitus. e Real-time PCR analysis of Kim-1 mRNA expression showing that diabetic CRP Tg mice (black bars) had much greater expression of Kim-1 by glomerular and tubular

of diabetic complications [1-3], we examined whether the enhanced diabetic renal injury manifest in $C R P \mathrm{Tg}$ mice is associated with increased renal inflammation. Immnunohistochemistry and real-time PCR analysis revealed that compared with age-matched normal mice, diabetic Wt mice developed significant renal inflammation, including increased epithelial cells than did diabetic Wt mice (white bars). f Periodic acidSchiff's-stained sections and (g) quantitative analysis of mesangial expansion showing that diabetic CRP Tg mice had more severe histological damage than diabetic Wt mice. Damage included glomerular hypercellularity, extracellular matrix deposition and mononuclear cells infiltrating the tubulointerstitium. Each microphotograph $(\mathbf{c}, \mathbf{f})$ is representative of groups of eight or six mice, magnification $\times 400$. Bars $(\mathbf{b}, \mathbf{d}, \mathbf{e}, \mathbf{g})$ represent mean $\pm \mathrm{SEM}$ from groups of eight or six mice. ${ }^{*} p<0.05, * * p<0.01,{ }^{* * *} p<0.001$ vs week 0 or normal; ${ }^{\dagger \dagger} p<0.01,{ }^{\dagger \dagger} p<0.001$ vs diabetic Wt mice or as indicated

renal infiltration of $\mathrm{CD}^{+} \mathrm{T}$ cells and $\mathrm{F} 4 / 80+$ macrophages (electronic supplementary material [ESM] Fig. 1), and upregulation of pro-inflammatory cytokines including IL-1 $\beta$ (ESM Fig. 2a-c) and TNF $\alpha$ (Fig. 2a-c). All these inflammatory changes were significantly exacerbated in diabetic $C R P$ Tg mice (ESM Figs 1 and 2a-c, Fig. 2a-c). 
Fig. 2 Renal inflammation is enhanced in CRP Tg mice with diabetes. a Immunohistochemical staining and (b) quantitative analysis of TNF $\alpha$ in Wt (white bars) and Tg (black bars) mice. c Real-time PCR analysis of Tnf $\alpha$. Results show that TNF $\alpha$ protein levels and mRNA expression were enhanced in CRP Tg mice with diabetes. d Western blot and (e) semiquantitative analysis of phospho-NF-kB/p65 (p-p65). f Immunohistochemistry and semi-quantitative analysis of nuclear translocation of phospho-NF-kB/p65 (p-p65) in (g) glomeruli and (h) and tubulointerstitium. Results show that activation of NF- $\mathrm{kB} / 65$ was significantly increased in $C R P$ Tg mice with diabetes. Magnification $(\mathbf{a}, \mathbf{f}) \times 400$. Data

$(\mathbf{b}, \mathbf{c}, \mathbf{e}, \mathbf{g}, \mathbf{h})$ represent the mean \pm SEM for groups of eight or six animals. ${ }^{*} p<0.05$,

$* * p<0.01, * * * p<0.001$ vs nor$\mathrm{mal} ;{ }^{\dagger \dagger} p<0.01,{ }^{\dagger \dagger} p<0.001 \mathrm{vs}$ diabetic Wt mice. gcs, glomerular cross-section; STZ, streptozotocin a

\section{Normal}
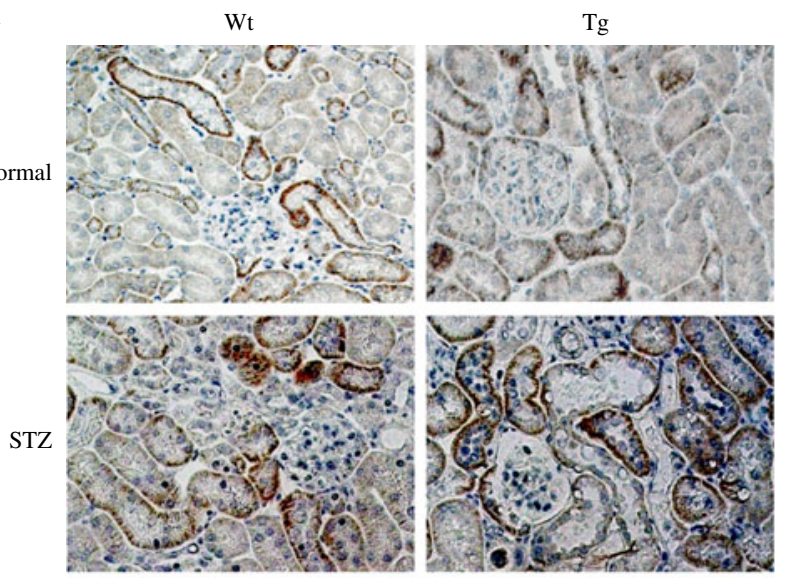

d

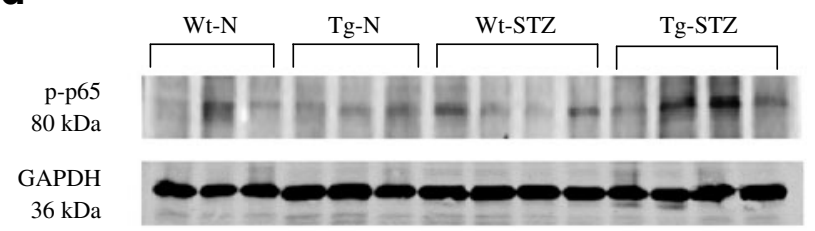

f

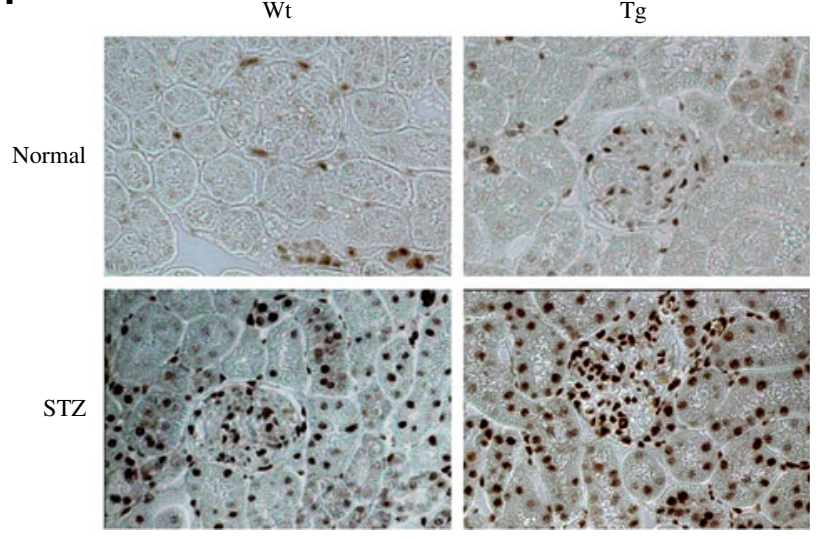

b
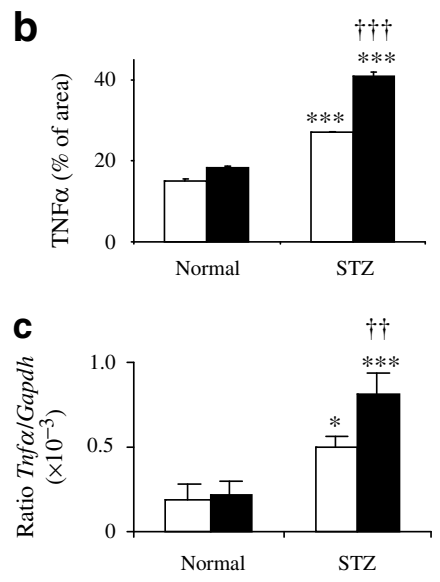

e

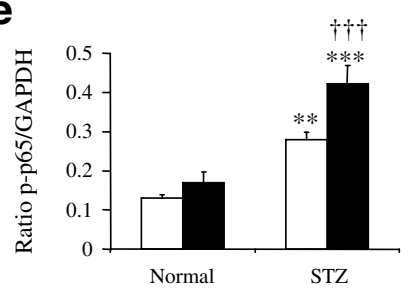

g

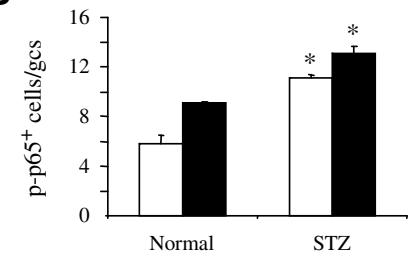

h

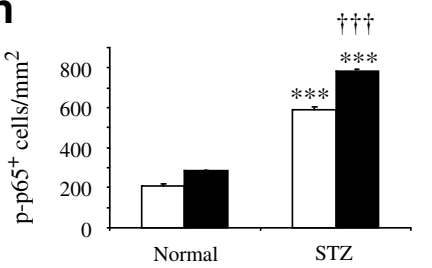

Renal fibrosis is enhanced in diabetic CRP Tg mice We next investigated whether increased renal fibrosis contributes to enhanced diabetic kidney injury in $C R P \mathrm{Tg}$ mice. As shown in Fig. 3 and ESM Fig. 2d-f, immunohistochemistry and real-time PCR analysis demonstrated that diabetic Wt mice developed significant collagen I, III and IV matrix accumulation and mRNA upregulation. All of these fibrotic changes were significantly enhanced in diabetic CRP Tg mice.

Enhanced CRP signalling and activation of $N F-\kappa B$ and TGF-B/SMAD3 signalling are a key mechanism by which CRP promotes diabetic renal injury Next, we investigated the signalling mechanisms that might underlie CRP exacerbation of diabetic renal injury. We first examined levels of the CRP receptor, CD32a. As shown in Fig. 4, a significant upregulation of CD32a was evident in normal $C R P \mathrm{Tg}$ mice compared with normal $\mathrm{Wt}$ mice. Likewise under diabetic conditions, CD32a abundance was significantly increased in diabetic $\mathrm{Wt}$ mice, presumably by glomerular and tubular epithelial cells; this increase was further aggravated in the diabetic kidney of CRP Tg mice, demonstrating enhanced CRP signalling in the diabetic kidney of that group.

Because NF- $\mathrm{kB}$ and TGF- $\beta / \mathrm{SMAD}$ signalling pathways are activated in the diabetic kidney and both have been shown to play a pathogenic role in the development of DKD [24-28], we examined whether enhanced diabetic kidney injury in CRP Tg mice is associated with enhanced activation of these two signalling pathways. Western blot analysis demonstrated that phosphorylation of NF-kB/p65 subunit was notably increased in the diabetic kidney of $\mathrm{Wt}$ 
Fig. 3 Renal fibrosis is enhanced in CRP Tg mice with diabetes. a Immunohistochemical staining and (b) quantitative analysis of collagen I (COL-I) in Wt (white bars) and Tg (black bars) mice. c Real-time PCR analysis of Col-I (also known as Collal) mRNA expression. d Immunohistochemical staining and (e) quantitative analysis of collagen IV (COL-IV). f Realtime PCR analysis of Col-IV (also known as Col4a4) mRNA expression. Results show that increased collagen I and IV protein and mRNA expression in diabetic Wt mice was further enhanced in CRP Tg mice. Magnification (a, d) $\times 200$. Data $(\mathbf{b}, \mathbf{c}, \mathbf{e}, \mathbf{f})$ represent the mean \pm SEM for groups of eight or six animals. ${ }^{*} p<0.05,{ }^{*} p<0.01$, $* * * p<0.001$ vs normal; ${ }^{\dagger} p<0.05$, ${ }^{\dagger \dagger} p<0.01,{ }^{\dagger \dagger} p<0.001$ vs diabetic Wt mice. STZ, streptozotocin

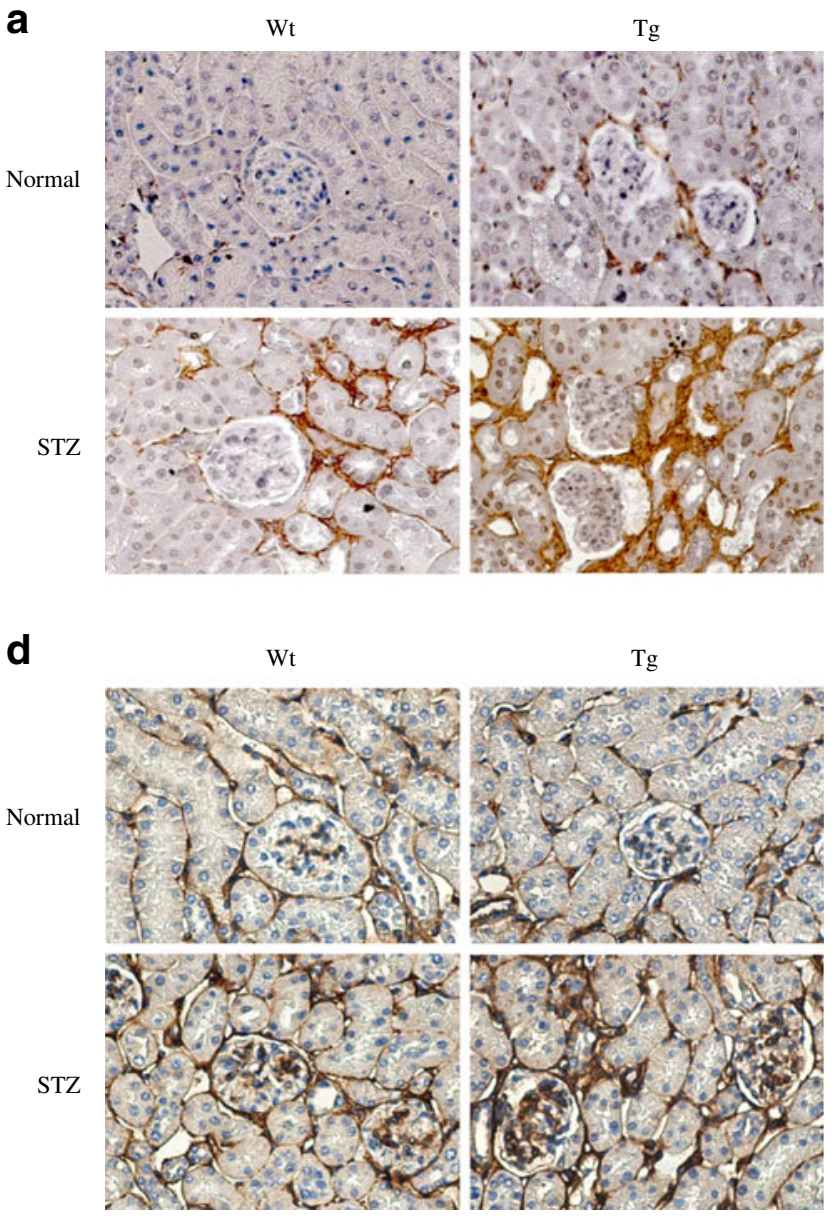

b
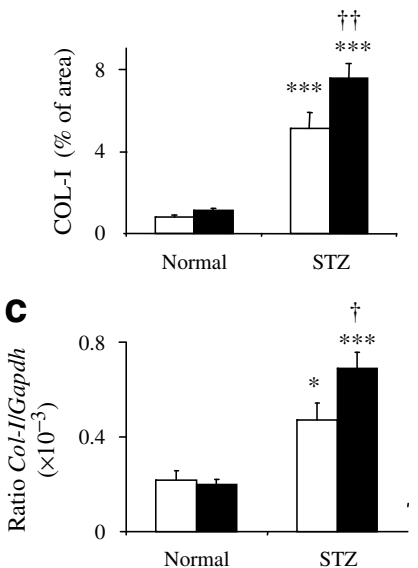

e
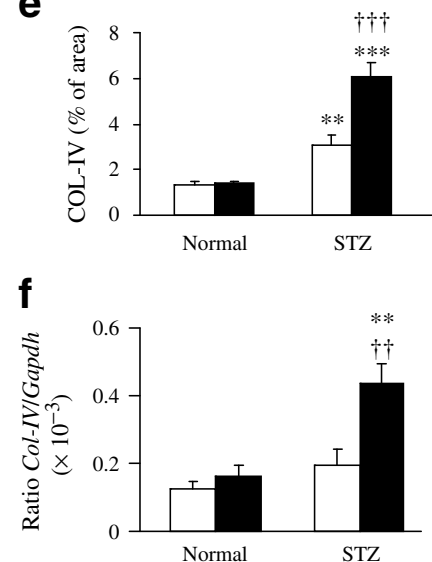

mice and even more increased in CRP Tg mice (Fig. 2d, e). Enhanced activation of NF- $\mathrm{KB}$ signalling in the diabetic kidney in $C R P \mathrm{Tg}$ mice was further evidenced by increased nuclear localisation of phosphorylated NF- $\mathrm{KB} / \mathrm{p} 65$ in the glomeruli and tubulointerstitium (Fig. 2f-h).

Enhanced renal fibrosis in CRP $\mathrm{Tg}$ mice was also associated with increased TGF- $\beta / \mathrm{SMAD}$ signalling in the diabetic kidney (Fig. 5). Real-time PCR and immunohistochemistry analysis detected higher levels of renal TGF- $\beta 1$ and CTGF protein and mRNA expression (Fig. 5a-f) in diabetic CRP $\mathrm{Tg}$ than in diabetic Wt mice. This was associated with enhanced SMAD signalling as demonstrated by higher levels of phosphorylated SMAD2/3 by western blot (Fig. $5 \mathrm{~g}, \mathrm{~h}$ ) and the enhancement of nuclear localisation of phosphorylated SMAD2/3 in many glomerular and tubulointerstitial cells (Fig. 5i-k).

CRP is induced by high glucose and signals through CD64 and CD32a to mediate renal inflammation and fibrosis directly and additively with high glucose in vitro To investigate the mechanism whereby CRP is elevated in patients with diabetes, we tested the hypothesis that CRP may be induced in human renal TEC (HK-2) under high glucose conditions. As shown in Fig. 6, real-time PCR and ELISA revealed that high glucose was able to induce CRP protein and mRNA expression. More importantly, although human TECs cultured with high glucose or CRP alone produced low-grade inflammatory and fibrotic responses as demonstrated by upregulation of pro-inflammatory cytokines (IL-1 $\beta, \mathrm{TNF} \alpha$ ) and pro-fibrotic growth factors (TGF$\beta 1$, CTGF), the combination of CRP and high glucose had an additive effect on upregulation of pro-inflammatory cytokines IL- $1 \beta$ and $\mathrm{TNF} \alpha$, and of pro-fibrotic growth factors TGF- $\beta 1$ and CTGF (Fig. 6c-f).

We then examined the mechanism by which CRP induces renal inflammation and fibrosis in vitro. It was apparent that CRP alone was able to upregulate proinflammatory cytokine (IL-1 $\beta, \mathrm{TNF} \alpha$ ) and pro-fibrotic growth factor (TGF- $\beta 1, \mathrm{CTGF}$ ) production by renal tubular epithelial cells (HK-2) in a time- and dosage-dependent manner, with production being increased at $3 \mathrm{~h}$ and peaking from 6 to $12 \mathrm{~h}$ (ESM Fig. 3). Monocyte chemoattractant protein-1 (MCP-1) abundance was also significantly increased in a dose-dependent manner after CRP stimulation, as shown in ESM Fig. 3e. Blockade of binding of human CRP to its receptors with neutralising antibodies to 
Fig. 4 CD32a abundance is enhanced in diabetic kidneys of CRP Tg mice. a Western blot and (b) semi-quantitative analysis of CD32a in Wt (white bars) and Tg (black bars) mice.

c Immunohistochemical analysis of CD32a; magnification $\times 400$. Results reveal that CD32a protein was significantly increased in the diabetic kidney of Wt mice and this was further enhanced in diabetic CRP Tg mice. Interestingly, compared with normal Wt mice, CD32a was readily upregulated in normal CRP Tg mice. Note that CD32a was largely expressed by glomerular and tubular epithelial cells. Each lane (a) or image (c) is representative of a group of eight mice; data (b) are expressed as mean \pm SEM for a group of eight mice. ${ }^{*} p<$ 0.05 vs normal; $\uparrow p<0.05$ vs $\mathrm{Wt}$ mice. STZ, streptozotocin

a

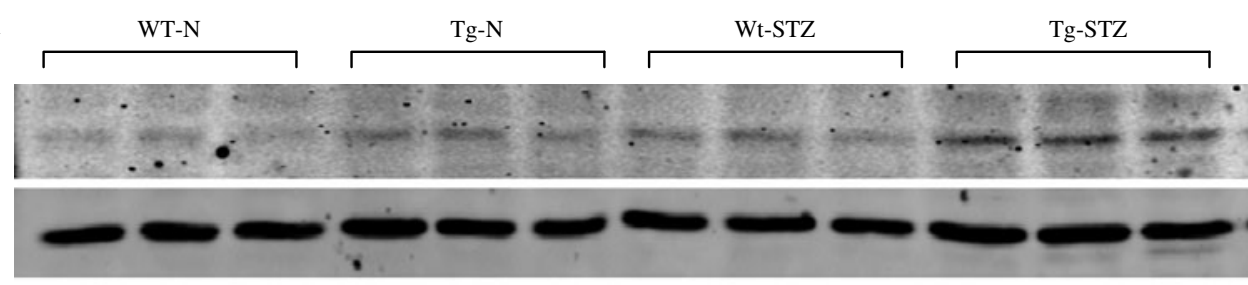

b

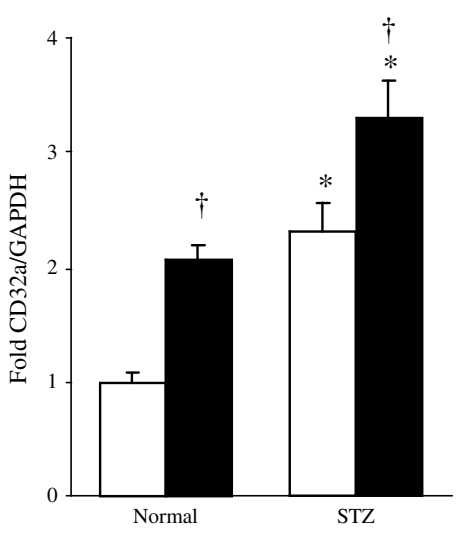

c

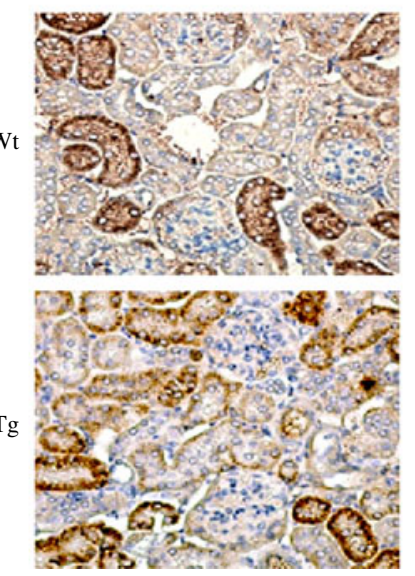

STZ

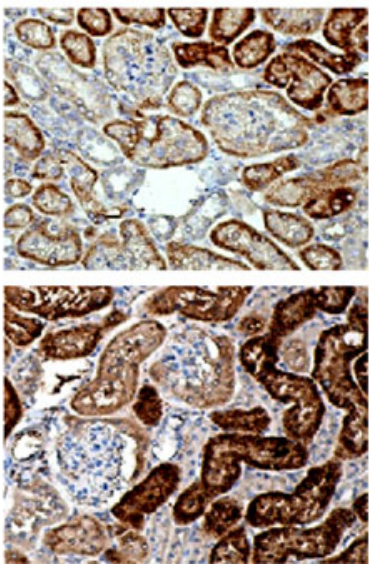

either CD64 or CD32a was able to partially abolish CRPstimulated expression of pro-inflammatory cytokines $(I l 1 b, \operatorname{Tnf} \alpha)$ and growth factors $(\operatorname{Tg} f b 1, \operatorname{Ctg} f)$ in human TECs (ESM Fig. 4). Interestingly, ELISA analysis revealed that addition of neutralising antibodies to CD64 or CD32a was also capable of inhibiting high glucoseinduced TNF $\alpha$ and TGF- $\beta 1$ production (ESM Fig. 5), suggesting a pathogenic importance for CRP signalling in the pathogenesis of renal inflammation and fibrosis under diabetic conditions.

\section{Discussion}

The present study provides direct biological evidence for the pathogenic importance of human CRP in DKD. We found that diabetic kidney injury was worsened in $C R P \mathrm{Tg}$ mice compared with $\mathrm{Wt}$, as demonstrated by a significant increase in UAE and Kim-1 expression. We also found that enhanced diabetic renal injury in $C R P \mathrm{Tg}$ mice was associated with a pronounced upregulation of renal inflammation and fibrosis, which is mediated through activation of TGF- $\beta / \mathrm{SMAD}$ and NF- $\mathrm{KB}$ signalling pathways. Together, these findings suggest that CRP may not only be a biomarker, but also a mediator in the development of DKD.

There are several mechanisms through which CRP may promote DKD. First, enhanced renal inflammation may be a mechanism by which CRP promotes diabetic kidney injury. It has been shown that the NF- $\mathrm{KB}$ signalling pathway is activated in human diabetic nephropathy and $\mathrm{CRP}$ is capable of inducing production of pro-inflammatory cytokines such as IL-1 $\beta$ and TNF $\alpha$ in cultured monocytes or endothelial cells via an NF- $\mathrm{kB}$-dependent mechanism [26, 29-32]. In the present study, we also demonstrated that renal inflammation, including IL- $1 \beta$ and TNF $\alpha$ production, and $\mathrm{CD}^{+} \mathrm{T}$ cell and $\mathrm{F} 4 / 80+$ macrophage infiltration, was significantly enhanced in the diabetic kidney of $C R P \mathrm{Tg}$ mice compared with diabetic Wt mice. In vitro, CRP alone induced production of pro-inflammatory cytokines such as IL- $1 \beta$ and TNF $\alpha$. We also found that CRP stimulates MCP1 production by HK-2 cells. These results are consistent with other studies in human endothelial cells and monocytes [33, 34]. More importantly, CRP itself was induced by high glucose, which, in turn, promoted high glucosemediated renal inflammation. This finding suggests that CRP may function as an inflammatory mediator or cofactor of high glucose to promote diabetic renal inflammation. Importantly, inhibition of high glucose-induced TNF $\alpha$ production by blockade of CRP signalling (with neutralising antibodies to $\mathrm{CD} 32 \mathrm{a}$ and $\mathrm{CD} 64$ ) provided additional evidence of the pathogenic activity of CRP in renal inflammation under diabetic conditions. Enhanced activation of the NF-KB signalling pathway in the diabetic kidney of $C R P \mathrm{Tg}$ mice, coupled with the ability of CD64- and CD32a-neutralising antibodies to block CRPinduced IL- $1 \beta$ and TNF $\alpha$ production, suggests that CRP probably acts via the CD64/CD32a-NF-KB pathway to exacerbate renal inflammation. This is consistent with previous reports [26, 31, 32]. 
Fig. 5 Mechanisms of enhanced renal fibrosis in $C R P$ $\mathrm{Tg}$ mice with diabetes. a Immunohistochemical staining and (b) quantitative analysis of TGF- $\beta 1$ in Wt (white bars) and $\mathrm{Tg}$ (black bars) mice. c Real-time PCR analysis of $T g f b 1$ mRNA expression. d Immunohistochemical staining and (e) quantitative analysis of CTGF. f Real-time PCR analysis of $\operatorname{Ctg} f$ mRNA expression. Results show that increased TGF- $\beta 1$ and CTGF protein levels and mRNA expression in diabetic Wt mice was further enhanced in CRP Tg mice. g Western blot analysis and (h) semi-quantitative data of phospho-SMAD2/3. i Immunohistochemistry and semi-quantitative data of phospho-SMAD2/3 (p-S2/3) nuclear translocation in (j) glomeruli and(k) tubulointerstitium. Results show that increased SMAD2/3 phosphorylation and nuclear translocation in diabetic $\mathrm{Wt}$ mice were further enhanced in diabetic kidneys of $C R P \mathrm{Tg}$ mice. Magnification (a, d, i) $\times 400$; data (b, c, e, f, h, $\mathbf{j}, \mathbf{k})$ represent the mean \pm SEM for groups of eight or six animals. ${ }^{*} p<0.05,{ }^{* *} p<0.01$, $* * * p<0.001$ versus normal; ${ }^{\dagger} p<0.05,{ }^{\dagger \dagger} p<0.01,{ }^{\dagger \dagger} p<0.001$ versus diabetic Wt mice. gcs, glomerular cross-section; STZ, streptozotocin a

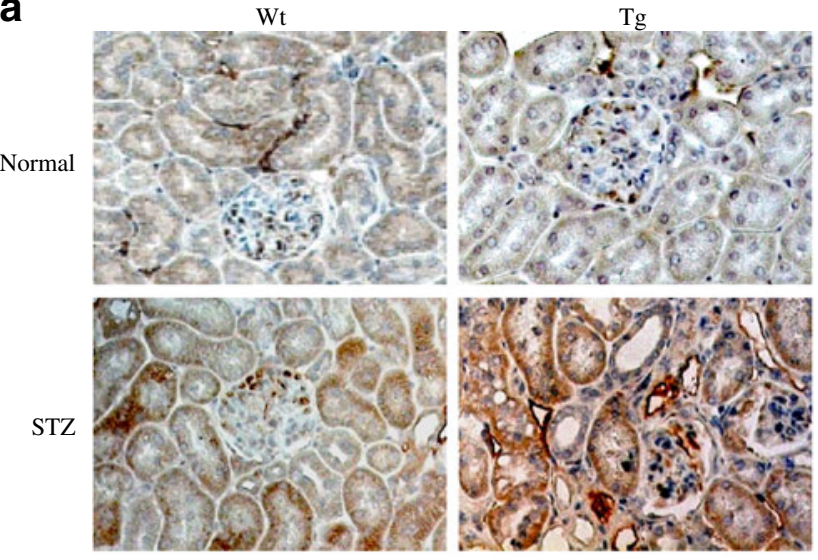

d

Wt
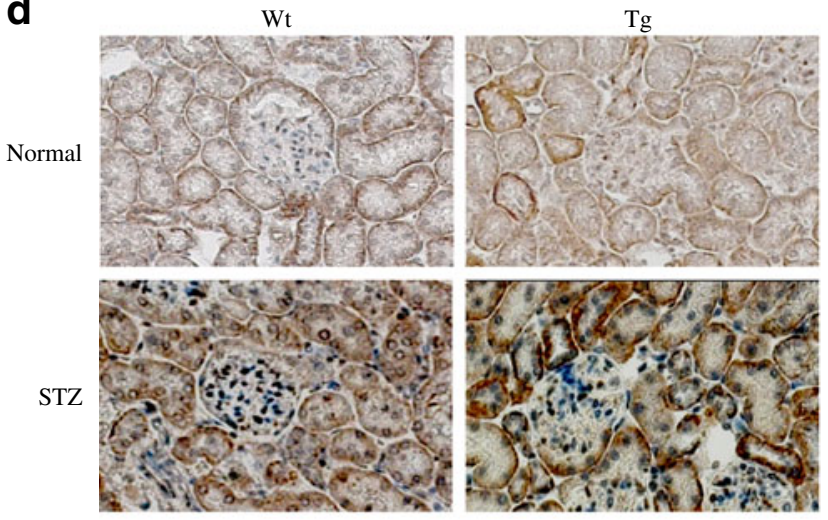

9

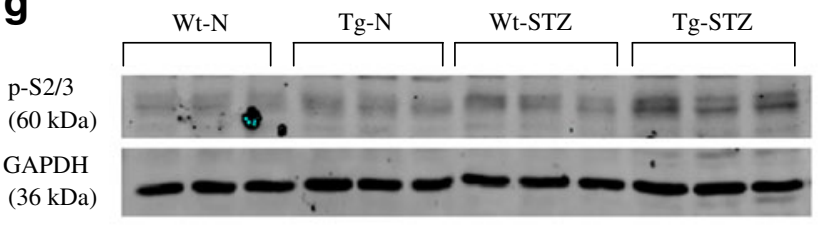

i

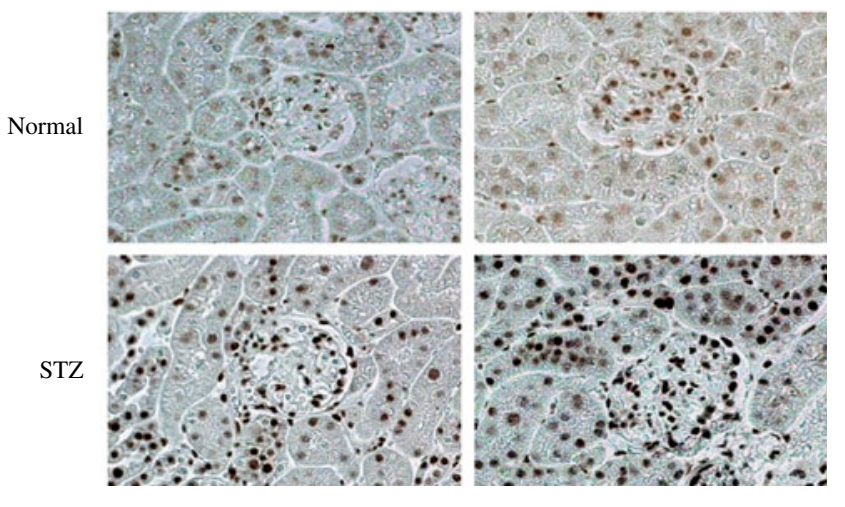

b
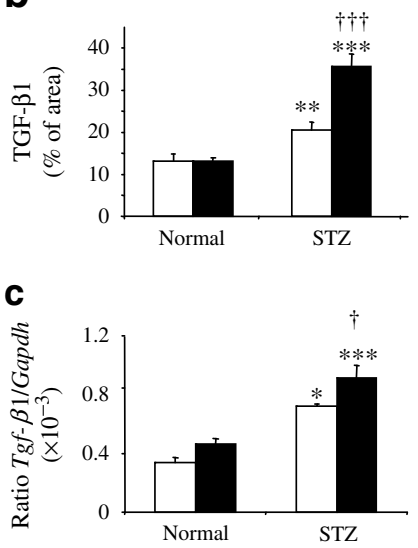

e

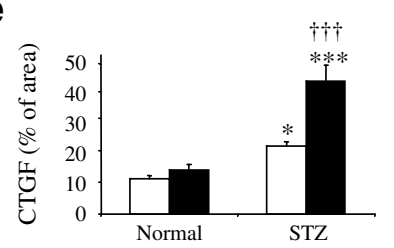

f

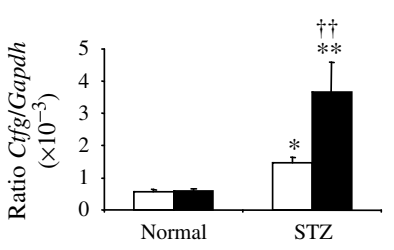

h

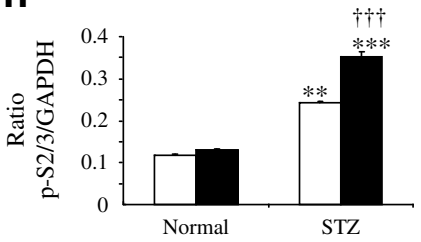

j

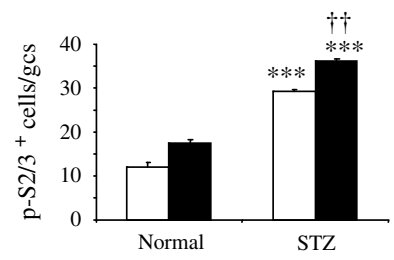

$\mathbf{k}$

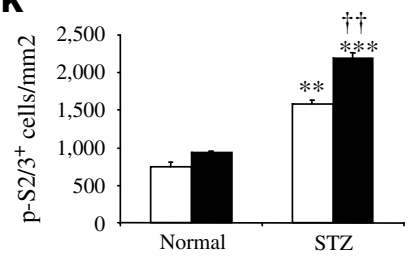

Enhancement of renal fibrosis in DKD might be achieved via increased TGF- $\beta /$ SMAD signalling. Renal fibrosis, characterised by increased accumulation of extracellular matrix within glomeruli and tubulointerstitium, is a hallmark of the final common pathway leading to loss of renal function associated with DKD. Activation of TGF- $\beta$ / SMAD signalling has been shown in experimental models and in human diabetic kidneys [24, 25, 27, 28], and is 

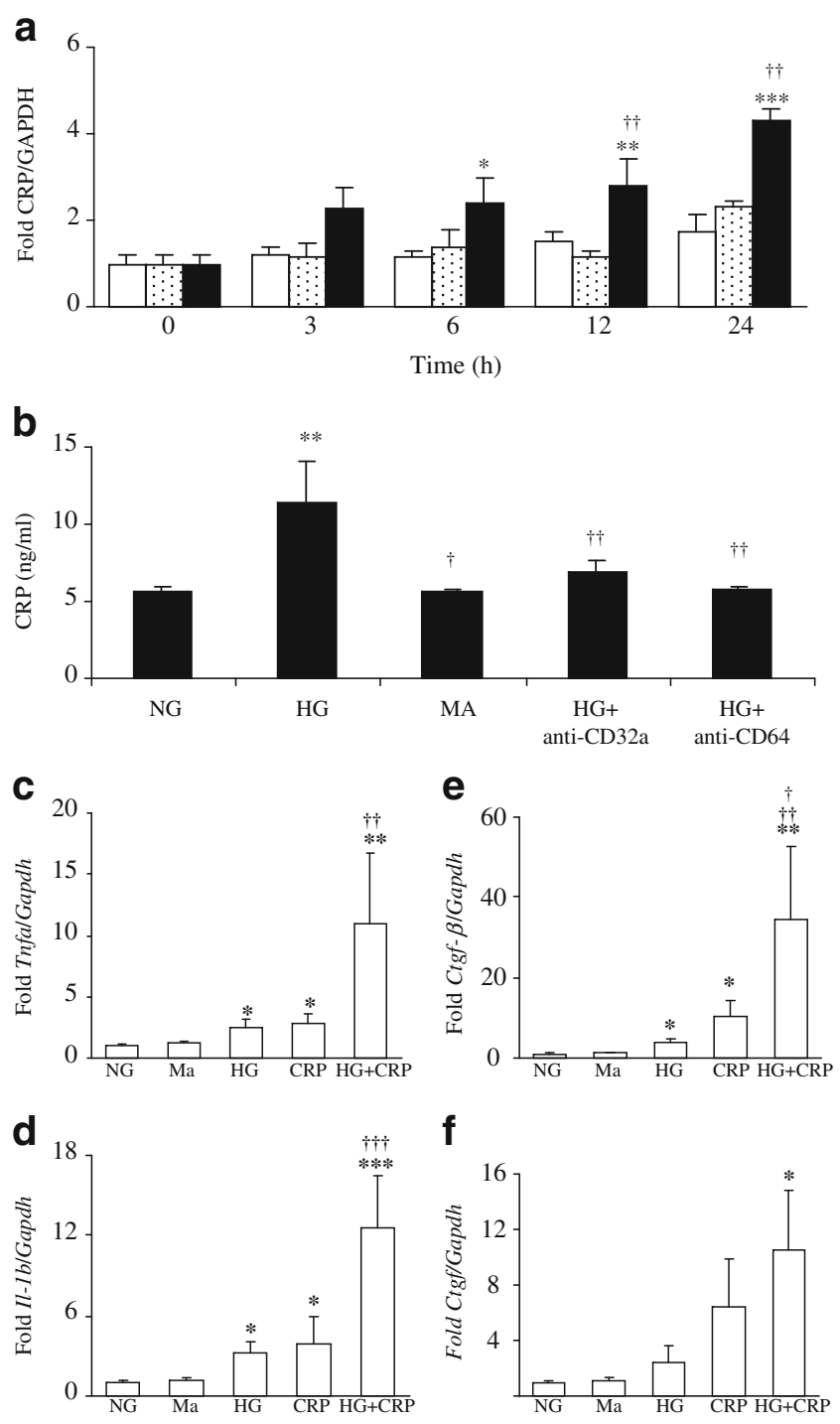

f

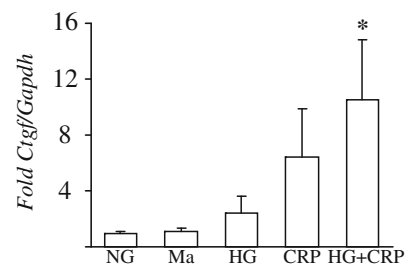

Fig. $6 \mathrm{CRP}$ is induced by high glucose, but promotes high glucosestimulated pro-inflammatory and pro-fibrotic responses in human TECs (HK-2). a High glucose (35 mmol/l) (HG, black bars), but not mannitol (35 mmol/l) (MA, dotted bars), induces CRP mRNA expression in HK-2 TECs in a time-dependent manner. NG, normal glucose. b ELISA revealed that addition of HG $(35 \mathrm{mmol} / \mathrm{l})$, but not mannitol $(35 \mathrm{mmol} / \mathrm{l})$, induced CRP protein production at $24 \mathrm{~h}$ after stimulation, a process that was blocked by addition of neutralising antibodies to CD32a and CD64. c Addition of HG (35 mmol/1) or CRP $(10 \mu \mathrm{g} / \mathrm{ml})$ alone, but not mannitol $(35 \mathrm{mmol} / \mathrm{l})$, was able to induce $\operatorname{Tn} f \alpha, I l l b(\mathbf{d}), \operatorname{Tg} f b 1$ (e) and $\operatorname{Ctg} f(\mathbf{f})$ mRNA expression, which was additively increased when combined together. Data represent the mean \pm SEM for at least three independent experiments. ${ }^{*} p<0.05$, ${ }^{* *} p<0.01,{ }^{* * *} p<0.001$ versus normal; ${ }^{\dagger} p<0.05,{ }^{\dagger \dagger} p<0.01,{ }^{\dagger \dagger} p<$ 0.001 versus $\mathrm{HG}$ or CRP alone

responsible for extracellular matrix production in vitro under high glucose and AGEs conditions [25, 35]. Thus, blockade of TGF- $\beta$ with neutralising anti-TGF- $\beta$ antibodies and targeting of the TGF- $\beta$ /SMAD signalling pathway by deletion of SMAD3 were able to inhibit diabetic kidney injury [27, 28, 36, 37]. A novel and significant finding in the present study was that CRP alone was able to induce pro-fibrotic growth factor production, including that of TGF- $\beta 1$ and CTGF, which was further enhanced in the presence of high glucose, indicating that CRP may act as a pro-fibrotic mediator that induces and promotes renal fibrosis under diabetic conditions. This may account for enhanced TGF- $\beta /$ SMAD signalling in the diabetic kidney of $C R P \mathrm{Tg}$ mice. The ability of neutralising antibodies to CD32a or CD64 to block CRP- or high glucose-induced renal TGF- $\beta$ as well as CTGF production revealed that CRP may bind $\mathrm{CD} 32 \mathrm{a}$ or $\mathrm{CD} 64$, thus mediating renal fibrosis through the TGF- $\beta$ signalling pathway under high glucose conditions.

Enhanced insulin resistance may be another plausible mechanism by which CRP worsens diabetic kidney injury. In patients with type 2 diabetes, for example, insulin resistance is associated with CRP [38]. This relationship holds in human $C R P \mathrm{Tg}$ mice, in which overexpression of human $C R P$ promotes the development of insulin resistance under high-fat diet conditions [39]. Similar results have also been obtained by other studies, showing that increased CRP causes insulin resistance by impairing skeletal muscle glucose uptake, which is mediated by Fc $\gamma$ receptor IIB [40]. Thus, it is likely that increased CRP and metabolic alterations may synergistically mediate diabetic kidney injury in CRP Tg mice, although this is not yet proven and further studies are warranted.

Finally, we found no evidence that hypertension developed in human CRP Tg mice under normal or diabetic conditions. This is consistent with previous reports that in human $C R P$ $\mathrm{Tg}$ mice atherosclerosis and ischaemia-induced cardiac remodelling can be worsened without alteration of blood pressure [17, 41]. This is further supported by our recent finding that human $C R P \mathrm{Tg}$ mice had normal blood pressure, but promoted angiotensin II-induced cardiac remodelling under hypertensive conditions similarly to that seen in littermate non-Tg mice [18]. In contrast, mice overexpressing rabbit $C R P$ and rats after adeno-associated virus-mediated human $C R P$ gene transfer did develop hypertension $[42,43]$. The mechanisms leading to discrepancy in the development of hypertension in animals that overexpress $C R P$ remain largely unclear. The question of whether the genetic variation in response to CRP between the species contributes to the development of hypertension can only be answered by further investigations.

In summary, CRP may exacerbate the kidney complications of diabetes by promoting renal inflammation and fibrosis, a process achieved via the $\mathrm{CD} 32 \mathrm{a}-$ and $\mathrm{CD} 64$-mediated activation of NF- $k B$ and TGF- $\beta /$ SMAD signalling pathways. These results suggest that targeted lowering of CRP might be of therapeutic benefit in DKD. 
Acknowledgements This work was supported by grants from the Research Grant Council of Hong Kong (GRF 768207 and 767508, and CUHK5/CRF09).

Contribution statement F.L. and H.Y.C. conceived experiments, analysed data and drafted the article. X.R.H and A.J.S. generated CRP $\mathrm{Tg}$ mice, conceived experiments and revised the manuscript. A.C.K. C., L Z. and P.F. analysed data and revised the manuscript. H.Y.L. designed, supervised and wrote the article. All authors approved the final version of the manuscript.

Duality of interest The authors declare that there is no duality of interest associated with this manuscript.

\section{References}

1. Navarro-Gonzalez JF, Mora-Fernandez C (2008) The role of inflammatory cytokines in diabetic nephropathy. $\mathrm{J}$ Am Soc Nephrol 19:433-442

2. Saraheimo M, Teppo AM, Forsblom C, Fagerudd J, Groop PH (2003) Diabetic nephropathy is associated with low-grade inflammation in type 1 diabetic patients. Diabetologia 46:1402-1407

3. Tuttle KR (2005) Linking metabolism and immunology: diabetic nephropathy is an inflammatory disease. J Am Soc Nephrol 16:1537-1538

4. Pradhan AD, Manson JE, Rifai N, Buring JE, Ridker PM (2001) C-reactive protein, interleukin 6, and risk of developing type 2 diabetes mellitus. JAMA 286:327-334

5. Schalkwijk CG, Poland DC, van Dijk W et al (1999) Plasma concentration of $\mathrm{C}$-reactive protein is increased in type I diabetic patients without clinical macroangiopathy and correlates with markers of endothelial dysfunction: evidence for chronic inflammation. Diabetologia 42:351-357

6. Pfutzner A, Forst T (2006) High-sensitivity C-reactive protein as cardiovascular risk marker in patients with diabetes mellitus. Diabetes Technol Ther 8:28-36

7. Ridker PM (2009) C-reactive protein: eighty years from discovery to emergence as a major risk marker for cardiovascular disease. Clin Chem 55:209-215

8. Abrahamian H, Endler G, Exner M et al (2007) Association of low-grade inflammation with nephropathy in type 2 diabetic patients: role of elevated CRP-levels and 2 different genepolymorphisms of proinflammatory cytokines. Exp Clin Endocrinol Diabetes 115:38-41

9. Stehouwer CD, Gall MA, Twisk JW, Knudsen E, Emeis JJ, Parving HH (2002) Increased urinary albumin excretion, endothelial dysfunction, and chronic low-grade inflammation in type 2 diabetes: progressive, interrelated, and independently associated with risk of death. Diabetes 51:1157-1165

10. Sabanayagam C, Lee J, Shankar A, Lim SC, Wong TY, Tai ES (2009) C-reactive protein and microalbuminuria in a multi-ethnic Asian population. Nephrol Dial Transplant 25:1167-1172

11. Goldberg RB (2009) Cytokine and cytokine-like inflammation markers, endothelial dysfunction, and imbalanced coagulation in development of diabetes and its complications. J Clin Endocrinol Metab 94:3171-3182

12. Mugabo Y, Li L, Renier G (2010) The connection between Creactive protein (CRP) and diabetic vasculopathy. Focus on preclinical findings. Current Diabetes Reviews 6:27-34

13. Pepys MB, Hirschfield GM, Tennent GA et al (2006) Targeting Creactive protein for the treatment of cardiovascular disease. Nature $440: 1217-1221$
14. Ridker PM (2009) The JUPITER trial: results, controversies, and implications for prevention. Circulation 2:279-285

15. Ridker PM, Danielson E, Fonseca FA et al (2008) Rosuvastatin to prevent vascular events in men and women with elevated Creactive protein. N Engl J Med 359:2195-2207

16. Danenberg HD, Szalai AJ, Swaminathan RV et al (2003) Increased thrombosis after arterial injury in human C-reactive proteintransgenic mice. Circulation 108:512-515

17. Paul A, Ko KW, Li L et al (2004) C-reactive protein accelerates the progression of atherosclerosis in apolipoprotein E-deficient mice. Circulation 109:647-655

18. Zhang R, Zhang YY, Huang XR et al (2010) C-reactive protein promotes cardiac fibrosis and inflammation in angiotensin IIinduced hypertensive cardiac disease. Hypertension 55:953-960

19. Brosius FC 3rd, Alpers CE, Bottinger EP et al (2009) Mouse models of diabetic nephropathy. J Am Soc Nephrol 20:2503-2512

20. Szalai AJ, McCrory MA (2002) Varied biologic functions of Creactive protein: lessons learned from transgenic mice. Immunologic research 26:279-287

21. Lan HY, Mu W, Nikolic-Paterson DJ, Atkins RC (1995) A novel, simple, reliable, and sensitive method for multiple immunoenzyme staining: use of microwave oven heating to block antibody crossreactivity and retrieve antigens. J Histochem Cytochem 43:97-102

22. Huang XR, Chung AC, Zhou L, Wang XJ, Lan HY (2008) Latent TGF-betal protects against crescentic glomerulonephritis. J Am Soc Nephrol 19:233-242

23. Chung AC, Huang XR, Zhou L, Heuchel R, Lai KN, Lan HY (2009) Disruption of the Smad7 gene promotes renal fibrosis and inflammation in unilateral ureteral obstruction (UUO) in mice. Nephrol Dial Transplant 24:1443-1454

24. Hong SW, Isono M, Chen S, Iglesias-De La Cruz MC, Han DC, Ziyadeh FN (2001) Increased glomerular and tubular expression of transforming growth factor-beta1, its type II receptor, and activation of the Smad signaling pathway in the $d b / d b$ mouse. Am J Pathol 158:1653-1663

25. Li JH, Huang XR, Zhu HJ et al (2004) Advanced glycation end products activate Smad signaling via TGF-beta-dependent and independent mechanisms: implications for diabetic renal and vascular disease. Faseb J 18:176-178

26. Mezzano S, Aros C, Droguett A et al (2004) NF-kappaB activation and overexpression of regulated genes in human diabetic nephropathy. Nephrol Dial Transplant 19:2505-2512

27. Fujimoto M, Maezawa Y, Yokote K et al (2003) Mice lacking Smad3 are protected against streptozotocin-induced diabetic glomerulopathy. Biochem Biophys Res Commun 305:1002-1007

28. Wang A, Ziyadeh FN, Lee EY et al (2007) Interference with TGFbeta signaling by Smad3-knockout in mice limits diabetic glomerulosclerosis without affecting albuminuria. Am J Physiol 293:F1657-F1665

29. Ballou SP, Lozanski G (1992) Induction of inflammatory cytokine release from cultured human monocytes by $\mathrm{C}$-reactive protein Cytokine 4:361-368

30. Hanriot D, Bello G, Ropars A et al (2008) C-reactive protein induces pro- and anti-inflammatory effects, including activation of the liver $\mathrm{X}$ receptor alpha, on human monocytes. Thromb Haemost 99:558-569

31. Liang YJ, Shyu KG, Wang BW, Lai LP (2006) C-reactive protein activates the nuclear factor-kappaB pathway and induces vascular cell adhesion molecule-1 expression through CD32 in human umbilical vein endothelial cells and aortic endothelial cells. J Mol Cell Cardiol 40:412-420

32. Mold C, Du Clos TW (2006) C-reactive protein increases cytokine responses to Streptococcus pneumoniae through interactions with Fc gamma receptors. J Immunol 176:7598-7604 
33. Guan H, Wang P, Hui R et al (2009) Adeno-associated virusmediated human $\mathrm{C}$-reactive protein gene delivery causes endothelial dysfunction and hypertension in rats. Clin Chem 55:274-284

34. Pasceri V, Chang J, Willerson J et al (2001) Modulation of Creactive protein-mediated monocyte chemoattractant protein-1 induction in human endothelial cells by anti-atherosclerosis drugs. Circulation 103:2531-2534

35. Li JH, Huang XR, Zhu HJ, Johnson R, Lan HY (2003) Role of TGF-beta signaling in extracellular matrix production under high glucose conditions. Kidney Int 63:2010-2019

36. Sharma K, Jin Y, Guo J, Ziyadeh FN (1996) Neutralization of TGF-beta by anti-TGF-beta antibody attenuates kidney hypertrophy and the enhanced extracellular matrix gene expression in STZ-induced diabetic mice. Diabetes 45:522-530

37. Ziyadeh FN, Hoffman BB, Han DC et al (2000) Long-term prevention of renal insufficiency, excess matrix gene expression, and glomerular mesangial matrix expansion by treatment with monoclonal antitransforming growth factor-beta antibody in $d b / d b$ diabetic mice. Proc Natl Acad Sci USA 97:8015-8020

38. Lu B, Yang Y, Yang Z et al (2010) Insulin resistance in Chinese patients with type 2 diabetes is associated with C-reactive protein independent of abdominal obesity. Cardiovasc Diabetol 9:92

39. Kaneko H, Anzai T, Nagai T et al. (2011) Human C-reactive protein exacerbates metabolic disorders in association with adipose tissue remodelling. Cardiovasc Res. doi:10.1093/cvr/ cvr088

40. Tanigaki K, Mineo C, Yuhanna IS et al (2009) C-reactive protein inhibits insulin activation of endothelial nitric oxide synthase via the immunoreceptor tyrosine-based inhibition motif of FcgammaRIIB and SHIP-1. Circ Res 104:1275-1282

41. Takahashi T, Anzai T, Kaneko H et al (2010) Increased C-reactive protein expression exacerbates left ventricular dysfunction and remodeling after myocardial infarction. Am J Physiol Heart Circ Physiol 299:H1795-H1804

42. Vongpatanasin W, Thomas GD, Schwartz R et al (2007) C-reactive protein causes downregulation of vascular angiotensin subtype 2 receptors and systolic hypertension in mice. Circulation 115:1020-1028

43. Han K, Hong KH, Park JH et al (2004) C-Reactive protein promotes monocyte chemoattractant protein-1-mediated chemotaxis through upregulating $\mathrm{CC}$ chemokine receptor 2 expression in human monocytes. Circulation 109:2566-2571 